\title{
Exact and explicit solutions to the $(3+1)$-dimensional Jimbo-Miwa equation via the Exp-function method
}

\author{
Turgut Öziş ${ }^{\mathrm{a}, *}$, İsmail Aslan ${ }^{\mathrm{b}}$ \\ a Department of Mathematics, Ege University, 35100 Bornova, Izmir, Turkey \\ b Department of Mathematics, Izmir Institute of Technology, 35430 Urla, Izmir, Turkey
}

\section{A R T I C L E I N F O}

\section{Article history:}

Received 2 September 2008

Received in revised form 8 October 2008

Accepted 8 October 2008

Available online 11 October 2008

Communicated by R. Wu

PACS:

02.30.Jr

04.20.Jb

05.45.Yv

Keywords:

Exp-function method

$(3+1)$-dimensional Jimbo-Miwa equation

Generalized solitary solutions

\begin{abstract}
A B S T R A C T
In this Letter, the Exp-function method, with the aid of a symbolic computation system such as Mathematica, is applied to the $(3+1)$-dimensional Jimbo-Miwa equation to show its effectiveness and reliability. Exact and explicit generalized solitary solutions are obtained in more general forms. The free parameters can be determined by initial or boundary conditions. Being less restrictive and concise, the method can be applied to many high-dimensional nonlinear evolution equations having wide applications in applied physical sciences.
\end{abstract}

(C) 2008 Elsevier B.V. All rights reserved.

\section{Introduction}

Many phenomena in physics and in the nonlinear sciences can be modeled by a class of integrable nonlinear evolution equations. Consequently, construction of traveling wave solutions of nonlinear equations plays an important role in the study of nonlinear phenomena. Nowadays, with the rapid development of software technology, solving nonlinear evolution equations via symbolic computation is taking an increasing role due to its efficiency, accuracy and its easy use. To this end, in the open literature, various methods have been presented in the last four decades. For example, Tanh-Coth function [1], Sine-Cosine function [2], Jacobi elliptic function method [3], symmetry method [4], Weierstrass function method [5], the F-expansion method [6], Homotopy perturbation method [7-11], variational iteration method [12] and so on. However, all methods mentioned above have some restrictions in their applications.

Recently, He and $\mathrm{Wu}$ [13] introduced a straightforward and concise method, called the Exp-function method, to obtain generalized solitary solutions and periodic solutions. The solution procedure of the method, with the aid of symbolic computation, is very simple and precise and can easily be extended to all kinds of nonlinear evolution equations. The Exp-function method has been successfully applied to various nonlinear evolution equations [14-23].

In our present work, by using the Exp-function method, we would like to construct some explicit and exact formal solutions for a well-known model, $(3+1)$-dimensional Jimbo-Miva equation [24-27], which is of particular interest in science and has the form

$$
u_{x x x y}+3 u_{y} u_{x x}+3 u_{x} u_{x y}+2 u_{y t}-3 u_{x z}=0
$$

where $u=u(x, y, z, t)$. Eq. (1) is firstly investigated by Jimbo and Miwa and its certain soliton solutions are obtained [28]. Then, it is studied by several authors regarding its solutions, symmetries and integrability properties [29-31].

Lately, Wazwaz [32,33] successfully studied one-soliton solutions to Eq. (1) by means of the tanh-coth method. He also employed the Hirota's bilinear method to the Jimbo-Miwa equation and confirmed that it is completely integrable and it admits multiple-soliton solutions of any order.

\footnotetext{
* Corresponding author. Tel.: +90 232388 1893; fax: +90 2323881036.

E-mail address: turgut.ozis@ege.edu.tr (T. Öziş).
} 


\section{The Exp-function method}

To start with, suppose we have a nonlinear partial differential equation for $u(x, t)$ in the form

$P\left(u, u_{x}, u_{t}, u_{x x}, u_{x t}, u_{t t}, \ldots\right)=0$,

where $P$ is a polynomial in its arguments. To determine $u(x, t)$ explicitly, we take the following six steps:

Step 1. Look for traveling wave solutions of Eq. (2) by introducing a complex variation $\xi=k x+w t$ and taking $u(x, t)=U(\xi)$, and transform it to the ordinary differential equation

$$
Q\left(U, U^{\prime}, U^{\prime \prime}, \ldots\right)=0 \text {, }
$$

where prime denotes the derivative with respect to $\xi$.

Step 2. If possible, integrate Eq. (3) term by term one or more times. This yields constant(s) of integration. For simplicity, the integration constant(s) can be set to zero.

Step 3. Assume that the solution $U(\xi)$ of Eq. (4) is in the form

$$
U(\xi)=\frac{a_{c} \exp (c \xi)+\cdots+a_{-d} \exp (-d \xi)}{b_{p} \exp (p \xi)+\cdots+b_{-q} \exp (-q \xi)},
$$

where $c, d, p$ and $q$ are unknown positive integers to be determined, and $a_{n}$ and $b_{m}$ are unknown constants.

Step 4. Determine the highest order nonlinear term and the linear term of highest order in Eq. (3) and express them in terms of (4). Then, in the resulting terms, balance the highest order Exp-function to determine $c$ and $p$, and the lowest order Exp-function to determine $d$ and $q$.

Step 5. Substitute (4) into Eq. (3) and equate the coefficients of $\exp (n \xi)$ to zero, obtain a system of algebraic equations for $a_{n}$, $b_{m}$, $k$ and $w$. Then, solve the system with the aid of a computer algebra system such as Mathematica, Maple or Matlab to determine these constants.

Step 6. Substitute the values in the previous step into expression (4) and find the traveling wave solutions of Eq. (2). Then, to check the correctness of the solutions, it is necessary to substitute them into the original Eq. (2).

\section{Solutions of the Jimbo-Miwa equation}

Now, to seek for the traveling wave solutions to Eq. (1), we make the transformation $u(x, y, z, t)=U(\xi), \xi=k x+m y+r z+w t$, where $k, m, r$ and $w$ are constants to be determined later. Then, we get

$$
k^{3} m U^{(4)}+6 k^{2} m U^{\prime} U^{\prime \prime}+(2 m w-3 k r) U^{\prime \prime}=0,
$$

where the primes and $U^{(i)}$ denote the derivatives with respect to $\xi$. Now, we make an ansatz

$$
U(\xi)=\frac{a_{c} \exp (c \xi)+\cdots+a_{-d} \exp (-d \xi)}{b_{p} \exp (p \xi)+\cdots+b_{-q} \exp (-q \xi)}
$$

for the solution of Eq. (5) and balance the terms $U^{\prime} U^{\prime \prime}$ and $U^{(4)}$. By simple calculation, we have

$$
U^{(4)}=\frac{k_{1} \exp [(c+15 p) \xi]+\cdots}{k_{2} \exp [16 p \xi]+\cdots}
$$

and

$$
U^{\prime} U^{\prime \prime}=\frac{k_{3} \exp [(2 c+4 p) \xi]+\cdots}{k_{4} \exp [6 p \xi]+\cdots}=\frac{k_{3} \exp [(2 c+14 p) \xi]+\cdots}{k_{4} \exp [16 p \xi]+\cdots},
$$

where $k_{i}$ 's are determined coefficients for simplicity. Balancing highest order of Exp-function in Eqs. (7) and (8), we have

$$
c+15 p=2 c+14 p,
$$

which leads to the result

$$
p=c \text {. }
$$

Similarly, from the ansatz (6), we have

$$
U^{(4)}=\frac{\cdots+l_{1} \exp [-(d+15 q) \xi]}{\cdots+l_{2} \exp [-16 q \xi]}
$$

and

$$
U^{\prime} U^{\prime \prime}=\frac{\cdots+l_{3} \exp [-(2 d+4 q) \xi]}{\cdots+l_{4} \exp [-6 q \xi]}=\frac{\cdots+l_{3} \exp [-(2 d+14 q) \xi]}{\cdots+l_{4} \exp [-16 q \xi]},
$$

where $l_{i}$ are determined coefficients for simplicity. Balancing lowest order of Exp-function in Eqs. (11) and (12), we have

$$
-(d+15 q)=-(2 d+14 q),
$$

which leads to the result

$$
q=d
$$


We can freely choose the values of $c$ and $d$ in general. However, the final solution does not strongly depend on the values of $c$ and $d$ [13].

Case 1: $p=c=1, d=q=1$.

In this case, the solution of Eq. (5) can be expressed as

$$
U(\xi)=\frac{a_{1} \exp (\xi)+a_{0}+a_{-1} \exp (-\xi)}{b_{1} \exp (\xi)+b_{0}+b_{-1} \exp (-\xi)} .
$$

Substituting (15) into Eq. (5), we have

$$
\begin{aligned}
& \frac{1}{A}\left[C_{4} \exp (4 \xi)+C_{3} \exp (3 \xi)+C_{2} \exp (2 \xi)+C_{1} \exp (\xi)+C_{0}+C_{-1} \exp (-\xi)\right. \\
& \left.\quad+C_{-2} \exp (-2 \xi)+C_{-3} \exp (-3 \xi)+C_{-4} \exp (-4 \xi)\right]=0
\end{aligned}
$$

where

$$
\begin{aligned}
A= & \left(b_{1} \exp (\xi)+b_{0}+b_{-1} \exp (-\xi)\right)^{5}, \\
C_{4}= & -k^{3} m a_{1} b_{0} b_{1}^{3}+3 k r a_{1} b_{0} b_{1}^{3}-2 m w a_{1} b_{0} b_{1}^{3}+k^{3} m a_{0} b_{1}^{4}-3 k r a_{0} b_{1}^{4}+2 m w a_{0} b_{1}^{4}, \\
C_{3}= & -6 k^{2} m a_{1}^{2} b_{0}^{2} b_{1}+12 k^{2} m a_{0} a_{1} b_{0} b_{1}^{2}+11 k^{2} m a_{1} b_{0}^{2} b_{1}^{2}+3 k r a_{1} b_{0}^{2} b_{1}^{2}-2 m w a_{1} b_{0}^{2} b_{1}^{2} \\
& -6 k^{2} m a_{0}^{2} b_{1}^{3}-16 k^{3} m a_{1} b_{-1} b_{1}^{3}+12 k r a_{1} b_{-1} b_{1}^{3}-8 m w a_{1} b_{-1} b_{1}^{3}-11 k^{3} m a_{0} b_{0} b_{1}^{3} \\
& -3 k r a_{0} b_{0} b_{1}^{3}+2 m w a_{0} b_{0} b_{1}^{3}+16 k^{3} m a_{-1} b_{1}^{4}-12 k r a_{-1} b_{1}^{4}+8 m w a_{-1} b_{1}^{4}, \\
\vdots & \\
C_{-4}= & k^{3} m a_{0} b_{-1}^{4}-3 k r a_{0} b_{-1}^{4}+2 m w a_{0} b_{-1}^{4}-k^{3} m a_{-1} b_{-1}^{3} b_{0}+3 k r a_{-1} b_{-1}^{3} b_{0}-2 m w a_{-1} b_{-1}^{3} b_{0} .
\end{aligned}
$$

Equating the coefficients of $\exp (j \xi)$ to zero and solving the resulting algebraic system for $a_{1}, a_{0}, a_{-1}, b_{1}, b_{0}, b_{-1}, k, m, r$ and $w$, we have the following sets of solutions:

First set:

$$
a_{0}=b_{0}\left(\frac{a_{1}}{b_{1}}-k\right) \mp \sqrt{k^{2} b_{0}^{2}-4 k^{2} b_{-1} b_{1}}, \quad a_{-1}=b_{-1}\left(\frac{a_{1}}{b_{1}}-2 k\right), \quad w=\frac{k\left(3 r-k^{2} m\right)}{2 m} .
$$

Second set:

$$
a_{0}=b_{0}\left(\frac{a_{-1}}{b_{-1}}+2 k\right), \quad a_{1}=0, \quad b_{1}=0, \quad w=\frac{k\left(3 r-k^{2} m\right)}{2 m} .
$$

Third set:

$$
a_{0}=0, \quad a_{-1}=b_{-1}\left(\frac{a_{1}}{b_{1}}-4 k\right), \quad b_{0}=0, \quad w=\frac{k\left(3 r-4 k^{2} m\right)}{2 m} .
$$

Now, substituting (17) into (15) yields the generalized solitary solution

$$
u(x, y, z, t)=\frac{a_{1} \exp (\xi)+b_{0}\left(\frac{a_{1}}{b_{1}}-k\right) \mp \sqrt{k^{2} b_{0}^{2}-4 k^{2} b_{-1} b_{1}}+b_{-1}\left(\frac{a_{1}}{b_{1}}-2 k\right) \exp (-\xi)}{b_{1} \exp (\xi)+b_{0}+b_{-1} \exp (-\xi)},
$$

where $\xi=k x+m y+r z+\frac{k\left(3 r-k^{2} m\right)}{2 m} t$. Next, substituting (18) into (15) leads to the following generalized solitary solution

$$
u(x, y, z, t)=\frac{b_{0}\left(\frac{a_{-1}}{b_{-1}}+2 k\right)+a_{-1} \exp (-\xi)}{b_{0}+b_{-1} \exp (-\xi)},
$$

where $\xi=k x+m y+r z+\frac{k\left(3 r-k^{2} m\right)}{2 m} t$. Finally, substituting (19) into (15) results in another generalized solitary solution, which reads

$$
u(x, y, z, t)=\frac{a_{1} \exp (\xi)+b_{-1}\left(\frac{a_{1}}{b_{1}}-4 k\right) \exp (-\xi)}{b_{1} \exp (\xi)+b_{-1} \exp (-\xi)},
$$

where $\xi=k x+m y+r z+\frac{k\left(3 r-4 k^{2} m\right)}{2 m} t$.

The free parameters in the obtained solutions might imply some meaningful results in the physical model.

Case 2: $p=c=2, d=q=1$.

Under such case, the trial function (6) can be expressed as follows

$$
U(\xi)=\frac{a_{2} \exp (2 \xi)+a_{1} \exp (\xi)+a_{0}+a_{-1} \exp (-\xi)}{\exp (2 \xi)+b_{1} \exp (\xi)+b_{0}+b_{-1} \exp (-\xi)},
$$

where we set the free parameter $b_{2}=1$ for simplicity. Substituting (23) into Eq. (5), we get

$$
\frac{1}{A}\left[C_{9} \exp (9 \xi)+C_{8} \exp (8 \xi)+\cdots+C_{1} \exp (\xi)+C_{0}+C_{-1} \exp (-\xi)+C_{-2} \exp (-2 \xi)+C_{-3} \exp (-3 \xi)+C_{-4} \exp (-4 \xi)\right]=0
$$


where $A=\left(\exp (2 \xi)+b_{1} \exp (\xi)+b_{0}+b_{-1} \exp (-\xi)\right)^{5}$. Here, to save space, we omit to display the coefficients $C_{j}$ explicitly. Then, equating the coefficients of $\exp (j \xi)$ to zero and solving the resulting algebraic system for $a_{2}, a_{1}, a_{0}, a_{-1}, b_{1}, b_{0}, b-1, k, m, r$ and $w$, we have the following sets of solutions:

First set:

$$
a_{0}=b_{0}\left(a_{2}-2 k\right), \quad a_{-1}=0, \quad a_{1}=b_{1}\left(a_{2}-k_{1}\right) \mp \sqrt{-4 k^{2} b_{0}+k^{2} b_{1}^{2}}, \quad b_{-1}=0, \quad w=\frac{k\left(3 r-k^{2} m\right)}{2 m} .
$$

Second set:

$$
a_{0}=0, \quad a_{1}=0, \quad a_{-1}=b_{-1}\left(a_{2}-6 k\right), \quad b_{0}=0, \quad b_{1}=0, \quad w=\frac{3 k\left(r-3 k^{2} m\right)}{2 m} .
$$

Third set:

$$
a_{0}=\left(a_{2}-4 k\right) b_{0}, \quad a_{1}=a_{2} b_{1}, \quad a_{-1}=\left(a_{2}-4 k\right) b_{0} b_{1}, \quad b_{-1}=b_{0} b_{1}, \quad w=\frac{k\left(3 r-4 k^{2} m\right)}{2 m} .
$$

Now, substituting (25) into (23) yields the generalized solitary solution

$$
u(x, y, z, t)=\frac{a_{2} \exp (\xi)+b_{1}\left(a_{2}-k_{1}\right) \mp \sqrt{-4 k^{2} b_{0}+k^{2} b_{1}^{2}}+b_{0}\left(a_{2}-2 k\right) \exp (-\xi)}{\exp (\xi)+b_{1}+b_{0} \exp (-\xi)},
$$

where $\xi=k x+m y+r z+\frac{k\left(3 r-k^{2} m\right)}{2 m} t$. Next, substituting (26) into (23) leads to the following generalized solitary solution

$$
u(x, y, z, t)=\frac{a_{2} \exp (2 \xi)+b_{-1}\left(a_{2}-6 k\right) \exp (-\xi)}{\exp (2 \xi)+b_{0} \exp (-\xi)},
$$

where $\xi=k x+m y+r z-\frac{3 k\left(r-3 k^{2} m\right)}{2 m} t$. Finally, substituting (27) into (23) results in another generalized solitary solution, which reads

$$
u(x, y, z, t)=\frac{a_{2} \exp (2 \xi)+a_{2} b_{1} \exp (\xi)+b_{0}\left(a_{2}-4 k\right)+b_{0} b_{1}\left(a_{2}-4 k\right) \exp (-\xi)}{\exp (2 \xi)+b_{1} \exp (\xi)+b_{0}+b_{0} b_{1} \exp (-\xi)},
$$

where $\xi=k x+m y+r z+\frac{k\left(3 r-4 k^{2} m\right)}{2 m} t$.

Moreover, we observe that $(28)$ is equivalent to (20).

Case 3: $p=c=2, d=q=2$.

Then the trial function (6) becomes

$$
U(\xi)=\frac{a_{2} \exp (2 \xi)+a_{1} \exp (\xi)+a_{0}+a_{-1} \exp (-\xi)+a_{-2} \exp (-2 \xi)}{b_{2} \exp (2 \xi)+b_{1} \exp (\xi)+b_{0}+b_{-1} \exp (-\xi)+b_{-2} \exp (-2 \xi)} .
$$

There are some free parameters in (31), so we set $b_{2}=1, b_{1}=0, b_{-1}=0$ for simplicity and thus (31) takes the form

$$
U(\xi)=\frac{a_{2} \exp (2 \xi)+a_{1} \exp (\xi)+a_{0}+a_{-1} \exp (-\xi)+a_{-2} \exp (-2 \xi)}{\exp (2 \xi)+b_{0}+b_{-2} \exp (-2 \xi)} .
$$

By the same procedure as illustrated in the previous cases, we obtain the following sets:

First set:

$$
a_{0}=b_{0}\left(a_{2}-2 k\right) \mp 2 \sqrt{k^{2} b_{0}^{2}-4 k^{2} b_{-2}}, \quad a_{-2}=\left(a_{2}-4 k\right) b_{-2}, \quad a_{-1}=0, \quad a_{1}=0, \quad w=\frac{k\left(3 r-4 k^{2} m\right)}{2 m} .
$$

Second set:

$$
a_{0}=0, \quad a_{-2}=\left(a_{2}-8 k\right) b_{-2}, \quad a_{-1}=0, \quad a_{1}=0, \quad b_{0}=0, \quad w=\frac{k\left(3 r-16 k^{2} m\right)}{2 m} .
$$

Now, substituting (33) into (32) yields the generalized solitary solution

$$
u(x, y, z, t)=\frac{a_{2} \exp (2 \xi)+b_{0}\left(a_{2}-2 k\right) \mp 2 \sqrt{k^{2} b_{0}^{2}-4 k^{2} b_{-2}}+b_{-2}\left(a_{2}-4 k\right) \exp (-2 \xi)}{\exp (2 \xi)+b_{0}+b_{-2} \exp (-2 \xi)},
$$

where $\xi=k x+m y+r z+\frac{k\left(3 r-4 k^{2} m\right)}{2 m} t$. Next, substituting (34) into (32) leads to the following generalized solitary solution

$$
u(x, y, z, t)=\frac{a_{2} \exp (2 \xi)+b_{-2}\left(a_{2}-8 k\right) \exp (-2 \xi)}{\exp (2 \xi)+b_{-2} \exp (-2 \xi)},
$$

where $\xi=k x+m y+r z+\frac{k\left(3 r-16 k^{2} m\right)}{2 m} t$.

Case 4: $p=c=3, d=q=2$.

Then the trial function (6) becomes

$$
U(\xi)=\frac{a_{3} \exp (3 \xi)+a_{2} \exp (2 \xi)+a_{1} \exp (\xi)+a_{0}+a_{-1} \exp (-\xi)+a_{-2} \exp (-2 \xi)}{b_{3} \exp (3 \xi)+b_{2} \exp (2 \xi)+b_{1} \exp (\xi)+b_{0}+b_{-1} \exp (-\xi)+b_{-2} \exp (-2 \xi)} .
$$

We rewrite (37) in the following form

$$
U(\xi)=\frac{a_{3} \exp (2 \xi)+a_{2} \exp (\xi)+a_{1}+a_{0} \exp (-\xi)+a_{-1} \exp (-2 \xi)+a_{-2} \exp (-3 \xi)}{b_{3} \exp (2 \xi)+b_{2} \exp (\xi)+b_{1}+b_{0} \exp (-\xi)+b_{-1} \exp (-2 \xi)+b_{-2} \exp (-3 \xi)} .
$$


If we take $a_{-2}=b_{-2}=0$ in (38), then we get the same form as (31). This means that the Case 4 is equivalent to the Case 3 . Similarly, taking $a_{-1}=b_{-1}=a_{-2}=b_{-2}=0$ and $b_{3}=1$ in (38) means that the Case 4 is also equivalent to the Case 2. Moreover, it is possible to rewrite (37) as in the form

$$
U(\xi)=\frac{a_{3} \exp (\xi)+a_{2}+a_{1} \exp (-\xi)+a_{0} \exp (-2 \xi)+a_{-1} \exp (-3 \xi)+a_{-2} \exp (-4 \xi)}{b_{3} \exp (\xi)+b_{2}+b_{1} \exp (-\xi)+b_{0} \exp (-2 \xi)+b_{-1} \exp (-3 \xi)+b_{-2} \exp (-4 \xi)}
$$

Now, if we take $a_{0}=b_{0}=a_{-1}=b_{-1}=a_{-2}=b_{-2}=0$ in (39), then we get the same form as (15). This means that the Case 4 is equivalent to the Case 1.

\section{Conclusion}

In summary, we have successfully applied the Exp-function method to the $(3+1)$-dimensional Jimbo-Miwa equation and constructed generalized solitary solutions with parameters. The free parameters, of course, might be related to initial or boundary conditions as well. We predict that these solutions will be of great importance for analyzing the nonlinear phenomena arising in applied physical sciences.

\section{Acknowledgements}

We would like to thank the referee for their useful and valuable comments.

\section{References}

[1] E.G. Fan, Phys. Lett. A 277 (2000) 212.

[2] A.M. Wazwaz, Appl. Math. Comput. 150 (2004) 365.

[3] G.T. Liu, T.Y. Fan, Phys. Lett. A 345 (2005) 161.

[4] M.H.M. Moussa, R.M. El Shikh, Physica A 371 (2006) 325.

[5] Z.Y. Yan, Chaos Solitons Fractals 21 (2004) 1013.

[6] M.A. Abdou, Chaos Solitons Fractals 31 (2007) 95.

[7] J.H. He, Comput. Methods Appl. Mech. Eng. 178 (1999) 257.

[8] J.H. He, Chaos Solitons Fractals 26 (2005) 695.

[9] T. Öziș, A. Yıldııım, Int. J. Nonlinear Sci. Numer. Simul. 8 (2) (2007) 239.

[10] T. Öziş, A. Yıldırım, Int. J. Nonlinear Sci. Numer. Simul. 8 (2) (2007) 243.

[11] A. Yıldırım, T. Öziş, Phys. Lett. A 369 (2007) 70.

[12] T. Öziş, A. Yıldırım, J. Sound Vib. 306 (2007) 372.

[13] J.H. He, X.H. Wu, Chaos Solitons Fractals 30 (2006) 700.

[14] J.H. He, L.N. Zhang, Phys. Lett. A 372 (2008) 1044.

[15] X.H. Wu, J.H. He, Comput. Math. Appl. 54 (2007) 966

[16] T. Öziş, C. Köroğlu, Phys. Lett. A 372 (2008) 3836.

[17] J.H. He, M.A. Abdou, Chaos Solitons Fractals 34 (2007) 1421.

[18] S.A. El-Wakil, M.A. Madkour, M.A. Abdou, Phys. Lett. A 369 (2007) 62.

[19] S. Zhang, Phys. Lett. A 365 (2007) 448.

[20] S. Zhang, Appl. Math. Comput. 199 (2008) 242.

[21] S.D. Zhu, Phys. Lett. A 372 (2008) 654.

[22] C.Q. Dai, X. Chen, S. Wu, Nonlinear Anal., doi:10.1016/j.na.2007.11.034.

[23] C. Dai, X. Cen, S. Wu, Chaos Solitons Fractals, doi:10.1016/j.chaos.2008.02.021.

[24] D. Wang, W. Sun, C. Kong, H. Zhang, Appl. Math. Comput. 189 (2007) 878.

[25] X.Y. Tang, Z.F. Liang, Phys. Lett. A 351 (2006) 398

[26] X.Q. Liu, S. Jiang, Appl. Math. Comput. 158 (2004) 177.

[27] X.B. Hu, D.L. Wang, H.W. Tam, W.M. Xue, Phys. Lett. A 262 (1999) 310.

[28] M. Jimbo, T. Miwa, Publ. Res. Inst. Math. Sci. 19 (1983) 943.

[29] W.X. Ma, J. Math. Phys. 40 (1999) 3685.

[30] B. Dorizzi, B. Grammaticos, A. Ramani, P. Winternitz, J. Math. Phys. 27 (1986) 2848.

[31] S.Y. Lou, J.P. Weng, J. Math. Phys. 36 (1995) 3492.

[32] A.M. Wazwaz, Appl. Math. Comput. 196 (2008) 363.

[33] A.M. Wazwaz, Appl. Math. Comput. 203 (2) (2008) 592. 